\title{
Identification of Stage-Related and Severity- RRelated Biomarkers and Exploration of Immune Landscape for Dengue by Comprehensive Analyses
}

Nan Xiong 1,2,3, Qiangming Sun 1,3, *

1 Institute of Medical Biology, Chinese academy of Medical Sciences, and Peking Union Medical College, Kunming 650031, PR China; qsun@imbcams.com.cn

2 Kunming Medical University, Kunming 650500, PR China; Love_XiongNan@163.com

3 Yunnan Key Laboratory of Vaccine Research \& Development on Severe Infectious Diseases, Kunming 650031, PR China; qsun@imbcams.com.cn

* Correspondence: qsun@imbcams.com.cn; Tel.: 0871-68335165

\begin{abstract}
:
Background: At present, there are still no specific therapeutic drugs and appropriate vaccines for Dengue. Therefore, it is important to explore distinct clinical diagnostic indicators.
\end{abstract}

Method: In this study, we combined differentially expressed genes (DEGs) analysis, weighted co-expression network analysis (WGCNA) and Receiver Operator Characteristic Curve (ROC) to screen a stable and robust biomarker with diagnosis value for Dengue patients. CIBERSORT was used to evaluate immune landscape of Dengue patients. Gene Ontology (GO) enrichment, Kyoto Encyclopedia of Genes and Genomes (KEGG) analysis and Gene set enrichment analysis (GSEA) were applied to explore potential functions of hub genes.

Results: $C D 38$ and Plasma cells have excellent AUC in distinguishing clinical stages for Dengue patients, and activated memory CD4+ T cells, and Monocytes have good AUC for this function. 
ZNF595 has acceptable AUC in discriminating DHF from DF in whole acute stages. Negative inhibition of viral replication based on GO, KEGG and GSEA analysis results, up-regulated autophagy genes and the impairing immune system are potential reasons resulting in dengue hemorrhagic fever (DHF).

Conclusions: $C D 38$, Plasma cells, activated memory CD4+ T cells and Monocytes can be used to distinguish clinical stages for dengue patients, and ZNF595 can be used to discriminate DHF from DF.

Keywords: DH, DHF, CD38, ZNF595 and Autophagy

\section{Introduction}

Dengue was listed by the World Health Organization (WHO) as one of the top ten global health threats announced at the beginning of 2019 [1]. In the past few decades, Dengue has become the fastest-growing mosquito borne disease in the world [2-4], seriously endangering human health $[5,6]$. Vaccine research and development continue to make progress [7-12], but the antibody-dependent enhancement (ADE) limits the effectiveness of vaccines [13-16]. Asymptomatic infections increase incidence of dengue $[17,18]$ and effective treatments have not been confirmed. Therefore, it's urgent to explore the pathogenic mechanism of dengue fever and screen out molecular markers for better diagnosis and treatment.

Autophagy, a catabolic process that degrades damaged or abnormal intracellular components to recover nutrients, is essential for maintaining cell and body homeostasis [19, 20], and benefit to proliferation and infection of the Dengue virus (DENV) [21-24]. In DENV-ADE infection, 
cross-reactive antibodies mediate infection by inducing autophagy related proteins, and then suppress the innate immunity mediated by the mitochondria antiviral protein (MAVS) [25]. Immune response affects directly or indirectly host response to DENV in varying degrees, including symptomatic infection, asymptomatic infection [26, 27], dengue shock syndrome (DSS) and dengue hemorrhagic fever (DHF) [28-30]. Therefore, it is essential to explore autophagy and immune response during Dengue infection.

Transcriptomics researches are beneficial to assist researchers in better understanding disease causes [31] and locating biomarkers [32-34]. Our precious transcriptomics researches contributed to understand viral evolution and its impact on pathogenicity and vaccine development of DENV [35-38]. However, studies [39-41] published focused on multi-gene researches, and single analytical method (differentially expressed genes (DEGs) analyses), and did not link genomics with immune landscapes. In this study, we used a combination of DEG analyses, WGCNA analyses and Receiver Operator Characteristic Curve (ROC) to identify, validate and test biomarkers with diagnostic value of stages and severity in independent datasets, and applied the CIBERSORT website to analyze immune landscape differences among three stages and between DHF and Dengue Fever (DF) and explore correlations between genes and immune cells. 


\section{Results}

Figure 1 shows illustration for this study.

Figure 1. Flow chart diagram.

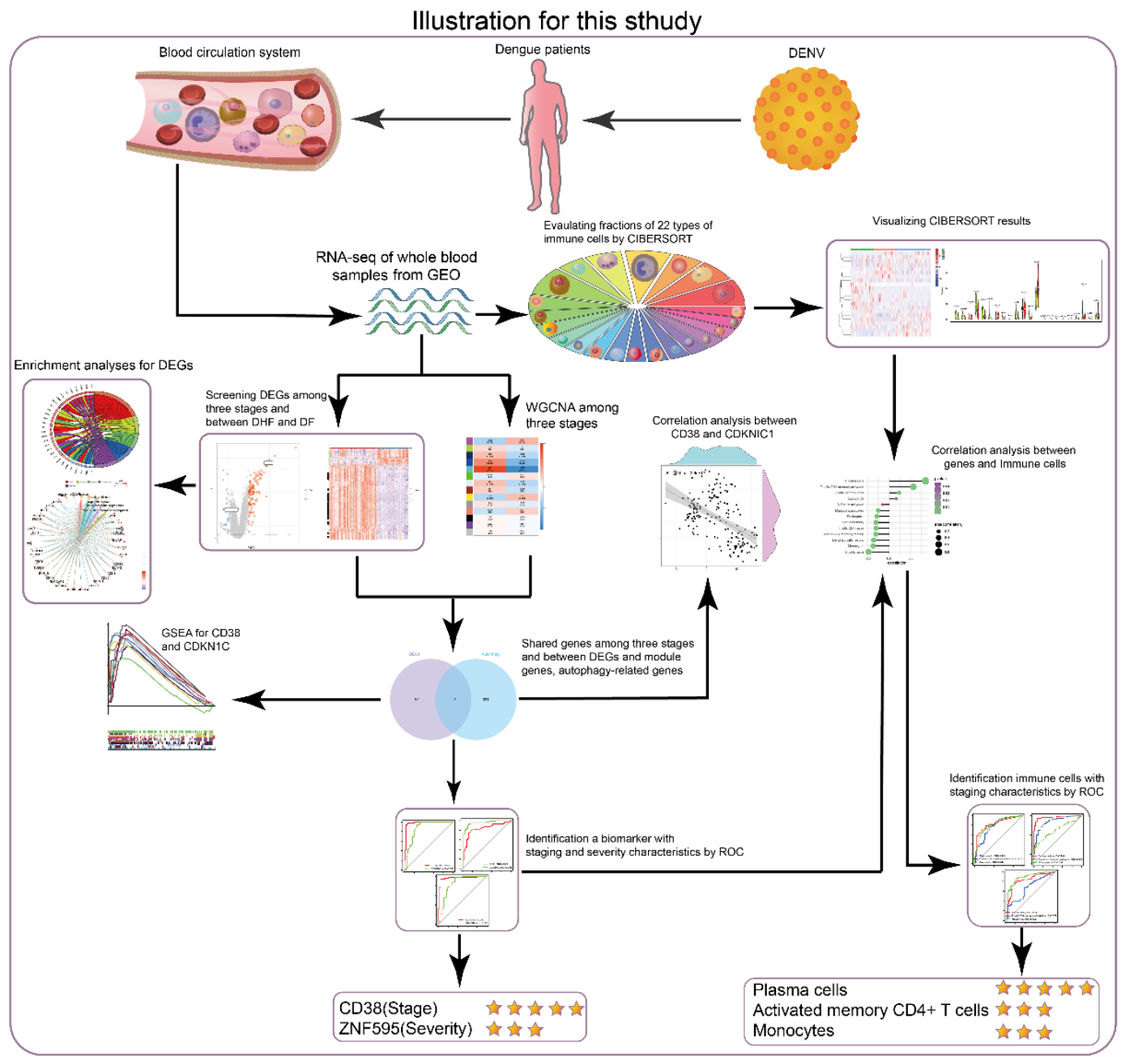

\subsection{Acquisition of DEGs and Mechanisms Analyses for Dengue}

The (Principal Component Analysis) PCA shows three stage samples can be distinguished

(Supplementary Figure S A-C). After normalizing the expression data from GSE43777 dataset on GLP201 platform, we identify 147 differentially expressed genes (DEGs, 121 up-regulated genes and 26 down-regulated genes) between the Convalescent (C) and the Early Acute (EA) stages (Figure 2A, 2F and Table S1), 124 DEGs (102 up-regulated genes and 22 down-regulated genes) between the C and the Late Acute (LA) stages (Figure 2B, 2G and Table S2) and 195 DEGs (74 up-regulated genes and 121 down-regulated genes) between the EA and LA stages 
(Figure 2C, 2H and Table S3) by a "Limma" package based on $|\mathrm{Log} 2 \mathrm{FC}| \geq 1$, and adjusted $\mathrm{P}$ value $<0.05$. Likewise, in the EA stage we obtained 30 DEGs (16 up-regulated genes and 14 down-regulated genes) (Figure 2D, 2I and Table S4) between dengue fever (DF) (n=15) and dengue hemorrhagic fever (DHF) (n=11) samples from GSE43777 dataset on GLP570 platform, and 58 DEGs (48 up-regulated genes and 10 down-regulated genes) (Figure 2E, 2J and Table S5) between DF ( $n=25)$ and DHF $(n=26)$ samples in the LA stage, based on $\mid$ Log2FC $\geq 1$ and P value $<0.05$

Gene Ontology (GO) analysis results show that these DEGs were mainly involved in potential biological processes (BP) (Figure 2K-2O) and pathways (Figure 2P-2T).

(GO) enrichment results (down-regulated genes in DHF samples enriched in negative regulation of viral replication) in Figure 2I and 2N caught our attention, because it suggested it was difficult to control viral replication in DHF patients in the EA stage. Relative studies[21, 24, 42] demonstrate Dengue Fever (DENV) inhibits cell apoptosis through autophagy, thereby promoting its replication. Therefore, we suspected related-autophagy genes may be activated in EA stage and LA stage. As the Supplementary Figure S J shown, in the LA stage, we identify definitely 1 different expression autophagy-related gene (CCL2) by intersecting DEGs between DHF and DH samples from GSE43777 analyzed on GLP570 platform with 232 autophagyrelated genes downloaded from Human Autophagy Database (HADb) (http://www.autophagy.lu/index.html). The boxplot diagram (Supplementary Figure S K) shows that the expression levels of CCL2 increases significantly in DHF samples in the LA stage, 
suggesting that DENV replicates more strongly in DHF than DF which may be one reason resulting in DHF in the LA stage. While we did not identify different expression of autophagyrelated genes between DF and DHF in the EA stage.

Figure 2. (A-E) Volcano maps, (F-J) heatmaps and (K-O) GO and (P-T) KEGG enrichment analyses for DEGs.

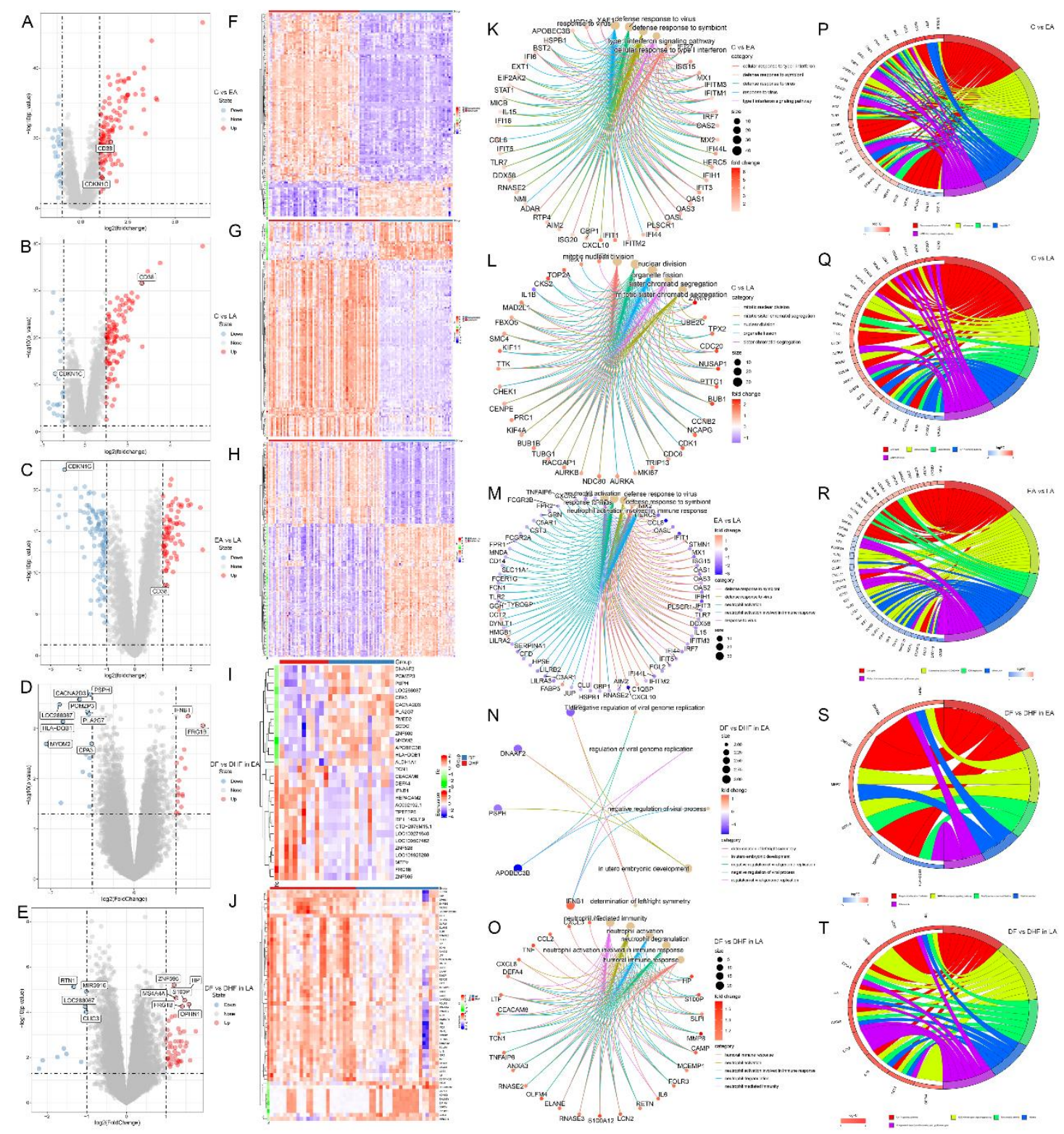




\subsection{Acquisition of Key Modules by weighted co-expression network analysis (WGCNA)}

Using the GSE43777 dataset analyzed on GLP201 platform, we performed a "WGCNA" package to obtain key modules associated with stage features of Dengue. We select 5, 4 and 12 (Figure 3A-3C) as the soft-threshold power respectively and 0.25 (Supplementary Figure S DF) as the cutoff height in the C vs EA group, in the C vs LA group and in the EA vs LA group. Modules consist of genes with similar expression properties (Supplementary Figure S G-I). In the cluster tree, we use each branch to represent a gene, and one color to show a co-expression module (Figure 3D-3F). The association of module eigengene (ME) values and Dengue stages was applied to assess the module-stage feature correlation.

WGCNA results show 14, 12 and 8 modules, respectively (Figure 3G-3I). In the C vs EA group, the turquoise module shows the most significant correction in Figure 3G, and In the C vs LA group the blue module shows the most significant correction in Figure 3H. Compared the EA with the LA group, the black module and the green module show the same highest correlation in Figure 3I. We regarded these modules mentioned above as key modules. 
Figure 3. Weighted co-expression network analysis. (A-C) The scale-free fit index (left) and average connectivity (right) of different soft threshold powers. (D-F) Clustering dendrograms of genes. (G-I) Heatmap of the module-trait displays correlations among different stages.

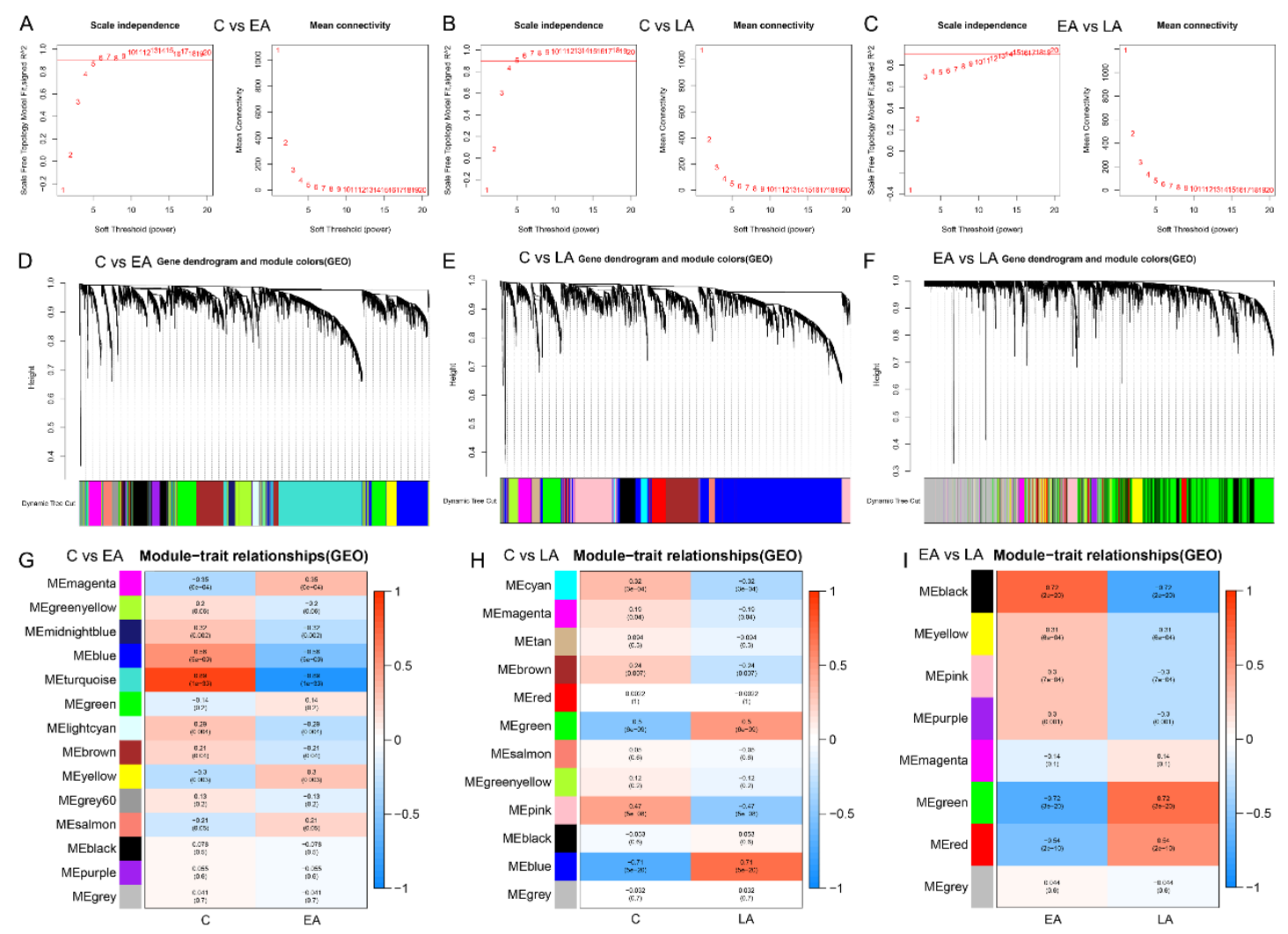

\subsection{Acquisition of Key Genes}

To filter reliable and strong hub genes (shared genes) accurately, Venn diagrams were used to visualize hub keys between DEGs and key modules by a website (http://www.ehbio.com/test/venn/\#/) [43]. The results illustrate 143, 99 and 187 hub genes by interacting the turquoise module with 147 DEGs (between the C and the EA stages) (Figure 4A), interacting the blue module with 124 DEGs (between the C and the LA stages) (Figure 4B), interacting the black and green modules with 195 DEGs (between the EA and LA stages) (Figure 4C), respectively. CD38 and CDKN1C were shared by the three phases (Figure 4D). The 
Figure 4G shows the expression levels of CD38 firstly increase and then decrease from day0 to the $C$ stage $\left.{ }^{* * *} \mathrm{P}<0.001\right)$ which differs from the expression level change of CDKN1C (Figure 4G). The Gene Set Enrichment Analysis (GSEA) show enrichment pathways of CD38 (Figure 4E) and CDKN1C (Figure 4F). Correlation analyses show in Figure 4H CD38 are negatively correlated with CDKN1C (spearman correlation= -0.5). We selected CD38 and CDKN1C for follow-up analyses.

Figure 4. (A-D) Venn diagrams screen out shared genes. (E and F) Showing the potential function enrichment pathways for CD38 and CDKN1C by GSEA. (G) The boxplot showing changes in expression levels of $C D 38$ and $C D K N 1 C$ over time. (H) A negative correlation between $C D 38$ and $C D K N 1 C$. 

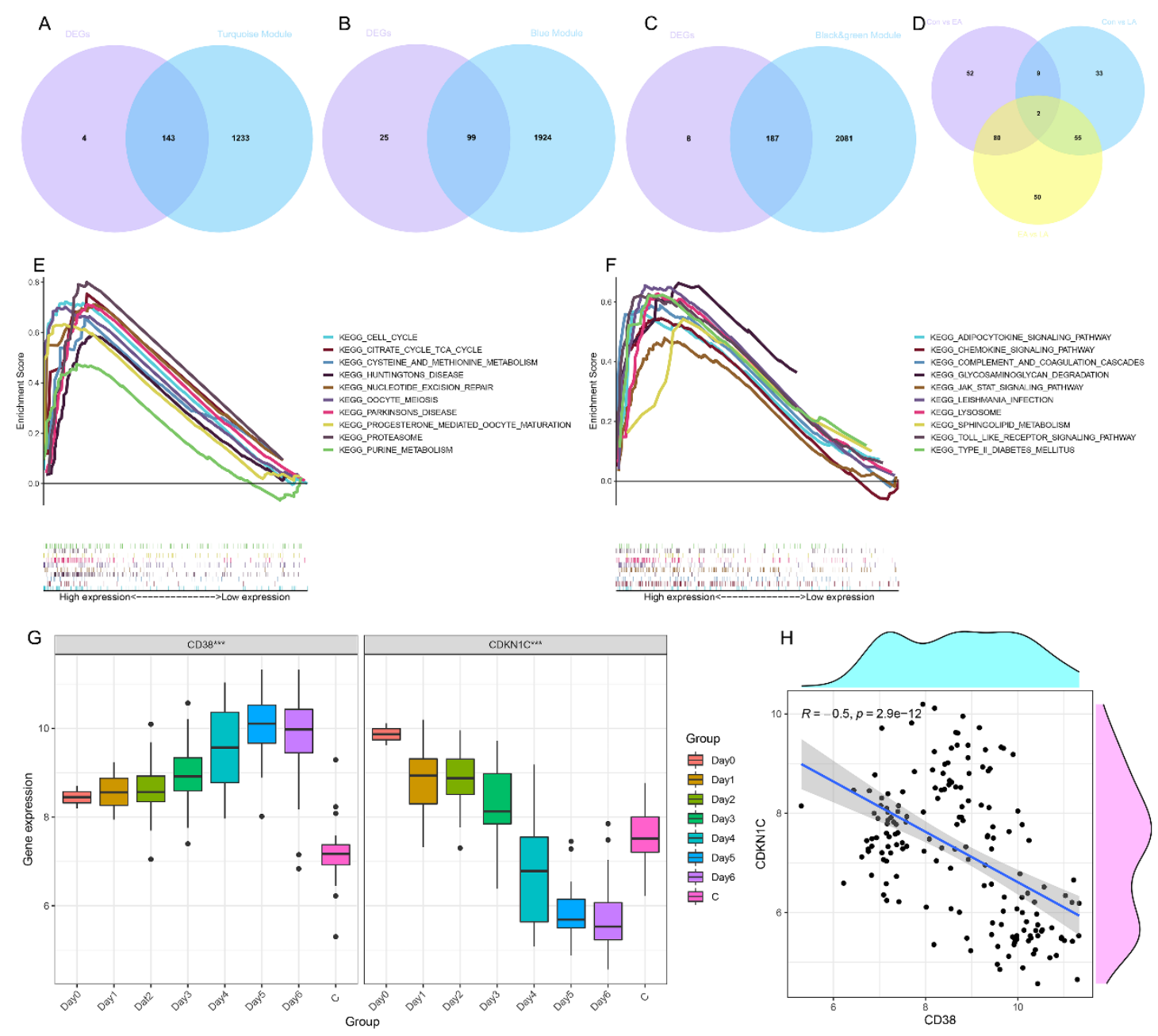

\subsection{Verification of Key Genes Value in Staging Diagnosis}

Because $C D 38$ and $C D K N 1 C$ express differently in three stages (Figure 5A-5B), we speculate that they can be used as biomarkers with staging characteristic to distinguish clinical stages for Dengue patients. To identify, verify and test the value of $C D 38$ and $C D K N 1 C$, we regarded GSE43777 datasets analyzed on the GPL201 platform (C stage, $n=48$; EA stage, $n=47$; LA stage, $\mathrm{n}=73$ ), GSE43777 datasets analyzed on the GPL570 platform (C stage, $n=24$; EA stage, $\mathrm{n}=26$; LA stage, $n=51$ ) and GSE28405 (C stage, $n=31$; EA stage, $n=57$; LA stage, $n=31$ ) as a training dataset, a verifying dataset and a testing dataset, respectively. 
In the training group, both CD38 and CDKN1C have excellent AUC (Area Under the Curve) in three comparing groups (Figure 5D-5F). However, in the verifying group, the staging diagnostic value of CDKN1C is not always good, like 0.575 of AUC in C vs EA group (Figure 5G). In the testing dataset, the staging diagnostic value of CD38 is still superior to CDKN1C (Figure 5J-5L). In addition, as Figure 5C shown, CD38 distinguishes perfectly Dengue samples from normal samples with an AUC of 100\% in GSE51808 dataset (9 normal samples and 28 Dengue samples). Therefore, CD38 can distinguish admirably different phases for Dengue patients and we selected CD38 as a biomarker for staging diagnosis of Dengue. There is no significant difference in expression levels of CD38 and CDKN1C between DH and DHF samples (Supplemental Figure S L), and among different serotypes in Dengue patients (Supplemental Figure S M). 
Figure 5. (A and B) Significantly different expression of CD38 and CDKN1C. (C) CD38 and CDKN1C can be used to distinguish Dengue samples from normal samples. Analyzing staging diagnosis value of CD38 and CDKN1C for Dengue by Area under curve (AUC): (D-F) Analyzed Firstly in the training group, (G-I) Subsequently verified in the verifying group, (J-L) Lastly tested in the testing group.
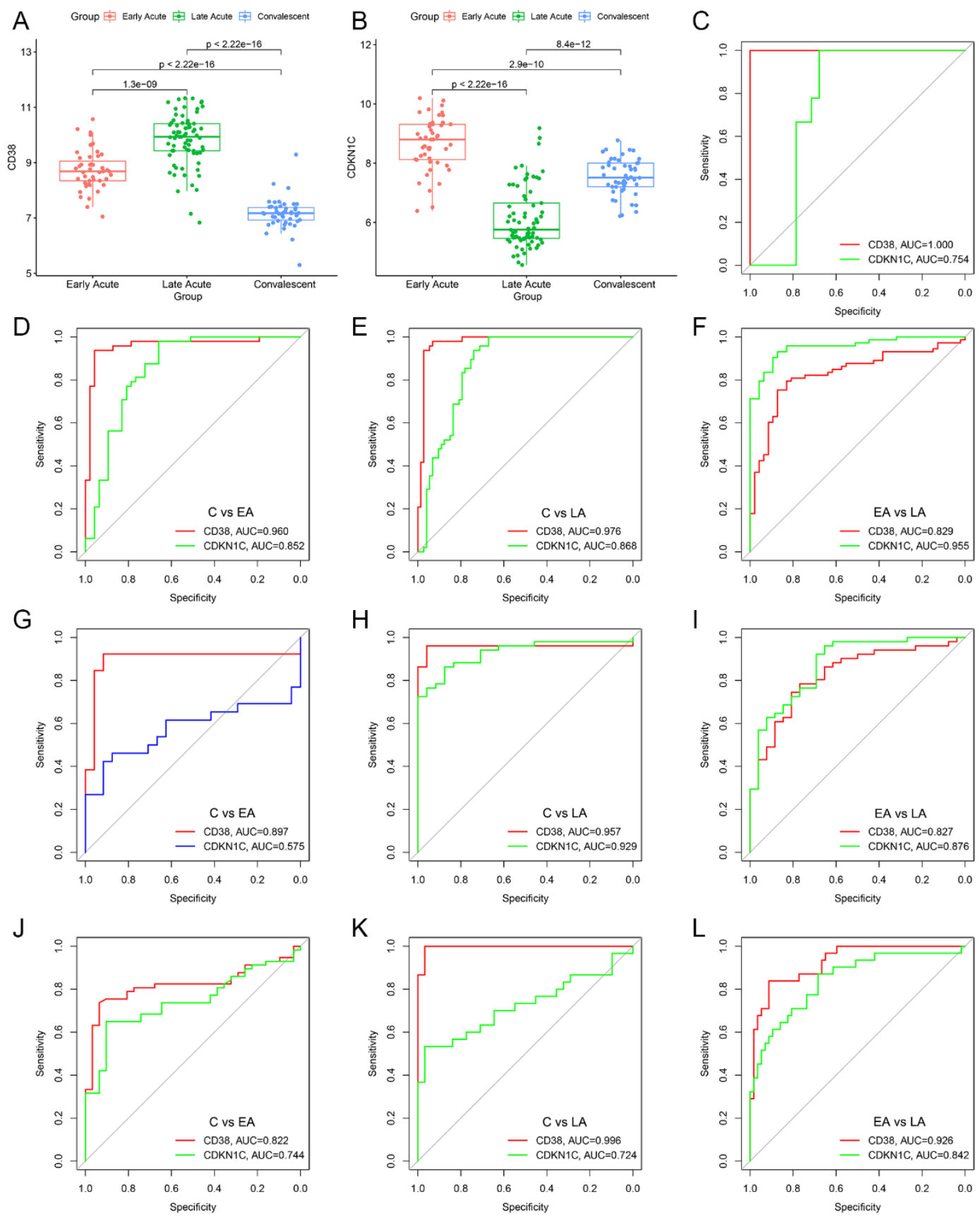


\subsection{Exploration of Biomarkers for DHF}

After intersecting 30 DEGs between DF ( $n=15)$ and DHF ( $n=11)$ samples from GSE43777 dataset on GLP570 platform in the EA stage with 58 DEGs between DF $(n=25)$ and DHF $(n=26)$ samples in the LA stage, we identify 6 shared DEGs (LOC101928288, TCN1, DEFA4, FRG1B, LOC286087 and ZNF595) (Figure 6A). We speculated that LOC101928288, TCN1, DEFA4, FRG1B, LOC286087 and ZNF595 can be used as biomarkers to discriminate DHF patients from DF patients due to different expression of them between DHF and DF. The GSE43777 datasets analyzed on the GPL570 platform in the EA stage (DF=15; DHF=11) and in the LA stage ( $\mathrm{DF}=25$; $\mathrm{DHF}=26$ ) were regarded as a training dataset; GSE43777 dataset analyzed on the GPL570 platform in Acute stage $(\mathrm{DF}=40$; $\mathrm{DHF}=37)$ was regarded as a verifying dataset; GSE51808 $(\mathrm{DF}=18 ; \mathrm{DHF}=10)$ and GSE18090 $(\mathrm{DF}=8 ; \mathrm{DHF}=10)$ datasets were regarded as testing datasets. The ROC (Receiver Operator Characteristic Curve) results (Figure 6B-6F) show ZNF595 always has acceptable diagnostic value in distinguishing DHF patients from DF patients. Therefore, ZNF595 was selected as a biomarker to predict DHF patients in Acute stages. 
Figure 6. (A) Shared DEGs between DF and DHF in the Acute stage. Analyzing diagnosis value of LOC101928288, TCN1, DEFA4, FRG1B, LOC286087 and ZNF595 for Dengue severity by Area under curve (AUC): (B-C) in the training dataset, (D) in the verifying dataset, (E-F) in testing datasets.
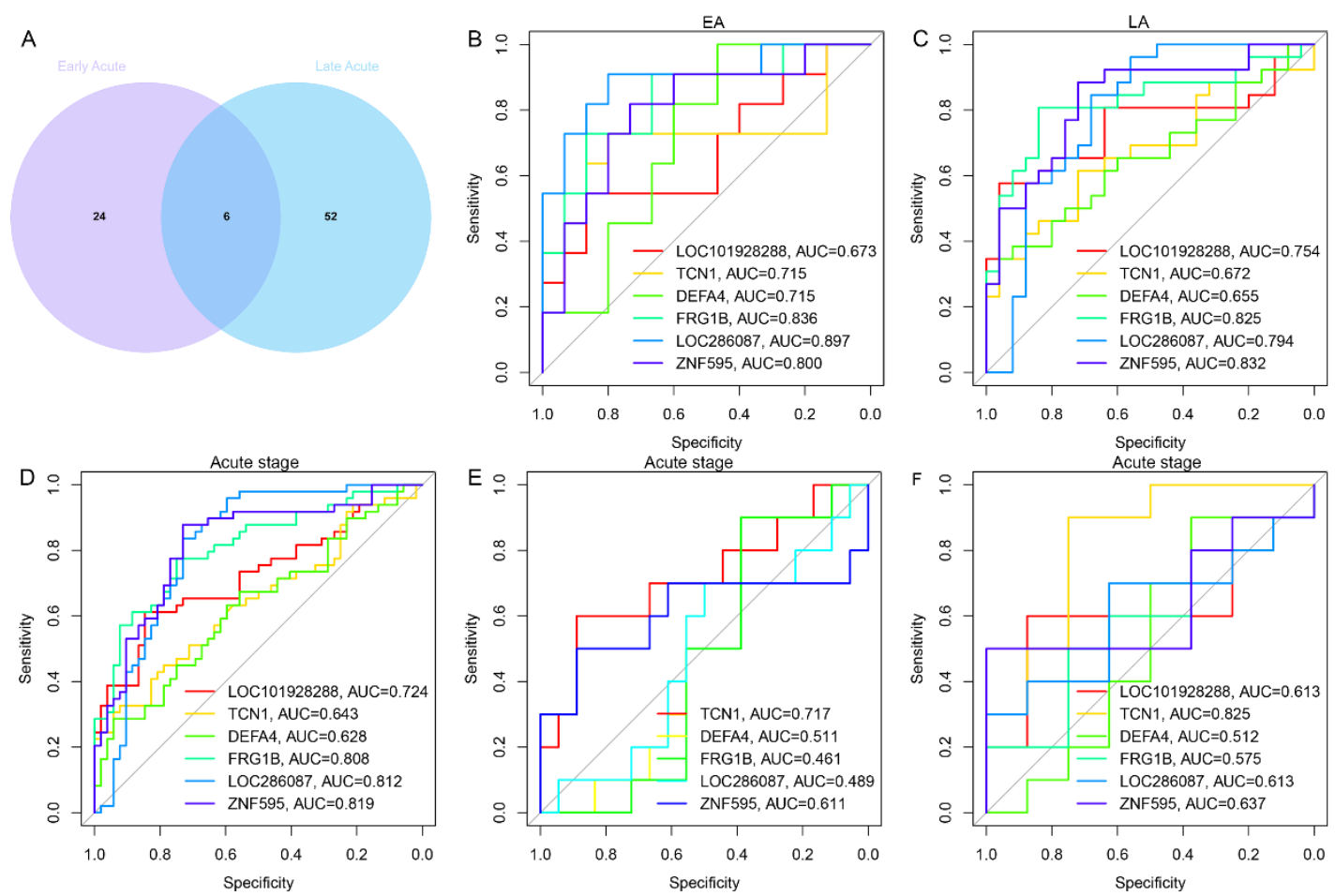

\subsection{Immune Landscape related to Characteristics of Dengue Patients}

GO enrichment analyses for 58 DEGs between DHF sample and DF showed neutrophil and humoral immune response were activated and KEGG pathway enrichment analyses show rich inflammatory pathways. Therefore, we analyzed fraction changes for 22 types of immune cells during Dengue infection by CIBERSORT. The GSE43777 dataset analyzed on the GLP201 platform was used to explore fractions of 22 types of immune cells in whole blood samples from Dengue patients in three phases and the GSE43777 dataset analyzed on the GLP570 platform was applied to explore differently infiltrating-immune characteristic between DF and 
DHF samples.

As Figure 7A-7C and 7F shown, in the EA phase, fractions of activated dendritic cells, Neutrophils, Monocytes, and M1 Macrophages $(\mathrm{P}<0.05)$ increase significantly compared to the LA and C phases. Compared the LA phase with the EA phase and the $C$ phase, fractions of Plasma cells and activated memory CD4+ T cells $(\mathrm{P}<0.05)$ increase clearly (Figure 7A-7C and 7F). While Compared with the EA phase and the LA phase, the increments in fractions of memory B cells, resting memory CD4+ T cells, resting dendritic cells, Eosinophils, and naïve B cells $(\mathrm{P}<0.05)$ are more obvious in the $\mathrm{C}$ phase (Figure 7A-7C and 7F). Analytical results above meant that immune cells with antigen presentation, phagocytosis and chemotaxis, immune cells involved in humoral immunity and memory cells were more obviously active in the EA, LA and $C$ phase, respectively.

In the LA stage, fractions of activated NK cells $(\mathrm{P}<0.05)$ increase in DHF samples (Figure 7E and $7 \mathrm{H})$, but fractions of CD8+T cells, gamma delta T cells, resting NK cells, M2 Macrophages and resting mast cells decrease (Figure 7E and 7H) $(\mathrm{P}<0.05)$, which imply immune response are damaged in DHF samples. In the EA stage, the immune response of DF patients is similar to that of DHF patients (Figure 7D and 7G). The immune response of DHF patients in the LA stage was significantly impaired which explained rapid deterioration of DHF patients in the LA stage. 
Figure 7. (A-E) Violin diagrams and (F-H) heatmaps display immune differences of immune cells in different comparing groups. (I-J) Correlation between genes (CD38 and ZNF595) and 22 types of immune cells (red rectangular: statistically significant $(\mathrm{P}<0.05)$ ).
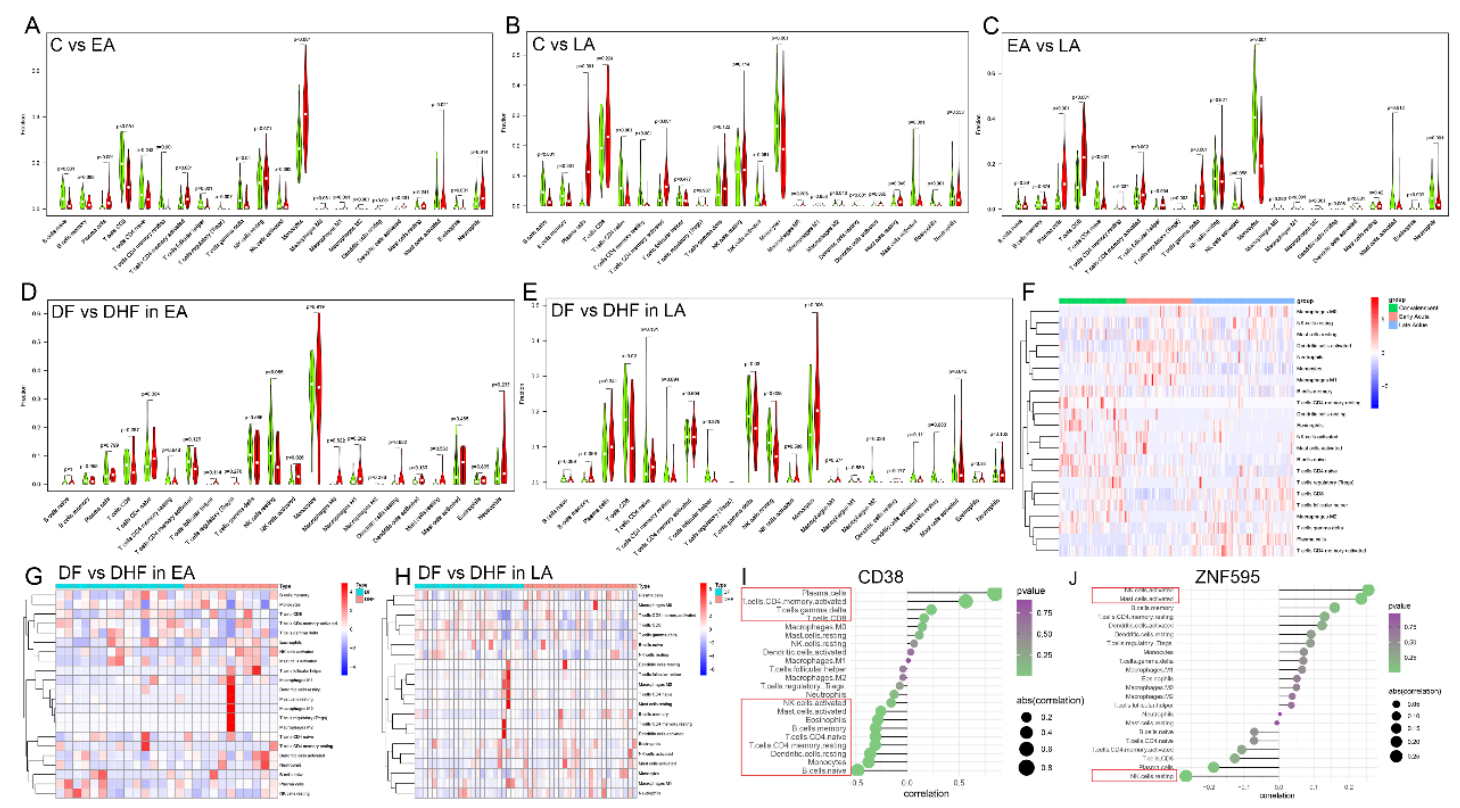

\subsection{Corrections between Gene and Immune Cells}

We analyze corrections between CD38 and immune cells in GSE43777 dataset (Figure 7I). The infiltration level of Plasma cells is the most positively related to CD38 (Pearson's correlation > 0.5) (Figure 7I). Therefore, the analytical results above show there is strong co-expression relationship between CD38 and plasma cells. However, correlation between immune cells and ZNF595 is not significant (Figure 7J). 


\subsection{Analyzing the Staging Characteristic of Immune Cells}

Because of high co-expression correction between CD38 and plasma cells, we speculated that infiltrating-immune plasma cells could also show distinct differences in three stages. To explore this characteristic, we regarded GSE43777 analyzed by GPL201 as a training set and GSE43777 analyzed by GPL570 as a test set. In the training group, the distinguishing value of Plasma cells, activated memory CD4+ T cells, and Monocytes in staging were good (Figure 8A8C) and Plasma cells have the highest distinguishing value. In the test group, we can obtain similar results in Figure 8D-8F. Plasma cells (AUC $=0.968$ ), activated memory CD4+ T cells (AUC $=0.845$ ) and Monocytes (AUC=0.869) can excellently distinguish Dengue samples from normal samples in GSE51808 (Figure 8G). Therefore, we can discriminate three stages based on the fraction of Plasma cells, activated memory CD4+ T cells and Monocytes in Dengue patients. 
Figure 8. Analyzing staging diagnosis value of immune cells for Dengue by AUC (A-C) in the training group and (D-F) in the test group. (G)Validating diagnosis value of immune cells between Dengue samples and normal samples.
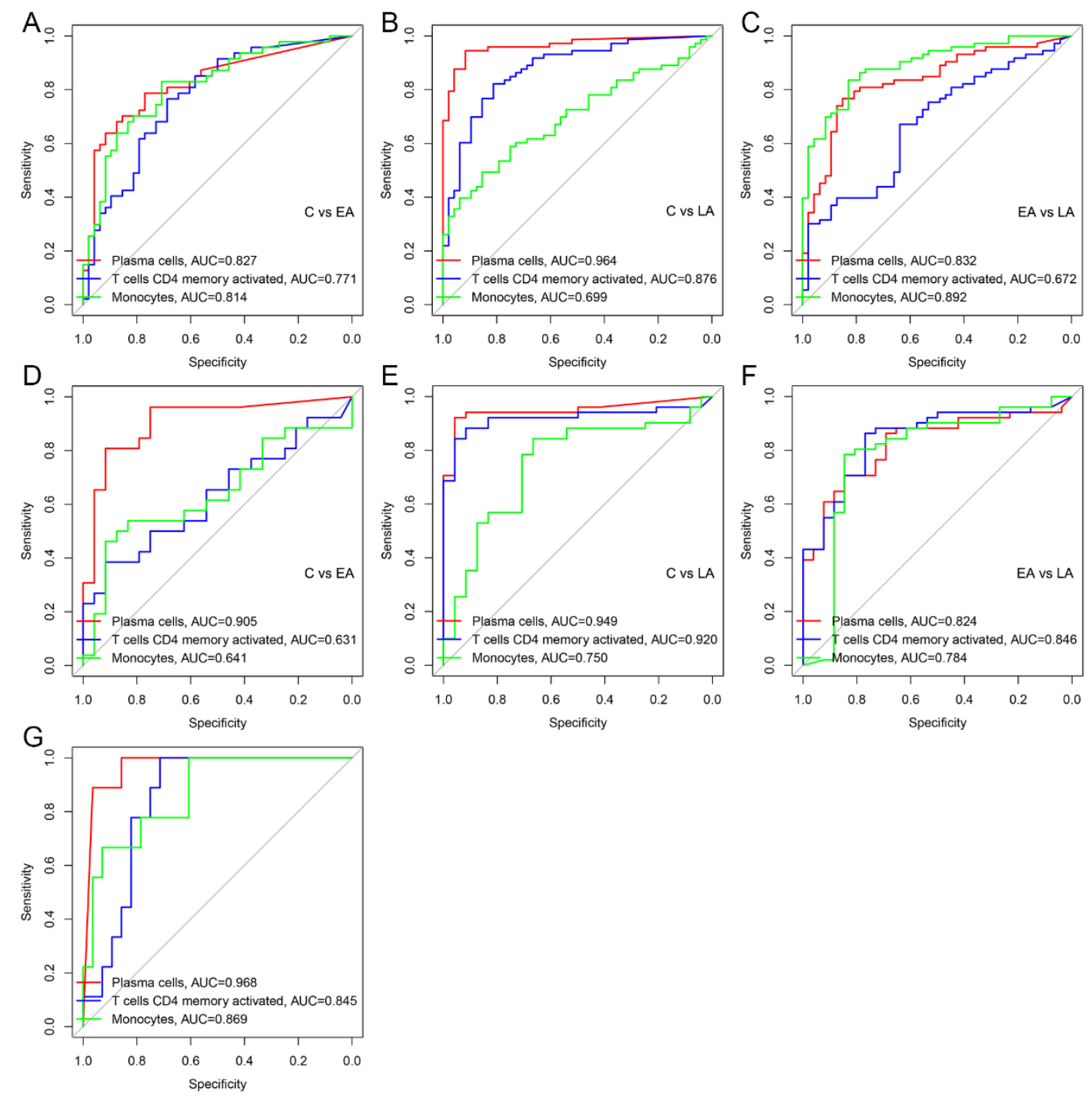

\section{Discussion}

CD38 which was identified, verified and tested in independent datasets could distinguish three clinical stages of Dengue and was significantly associated with plasma cells, but it could not use to predict severity which was similar to precious results [44] and distinguish different serotype. Similarly, ZNF595 can discriminate DHF from DF. Mechanistically, DEGs in the EA 
stage which inhibited viral replication were down-regulated in DHF, and a related-autophagy gene (CCL2) expressed differently and increased significantly in DHF, all of which suggested Dengue viruses (DENV) regulated autophagy to lead to DHF.

In addition, we analyzed systematically immune differences among three stages and between DHF and DF in the acute stage, and correlation between immune cells and genes. Increasing immune response eliminated DENV which avoided Dengue symptoms [27] and immune response kinetics dependent on initial lymphocyte numbers, were observed to correspond with illness severity [45]. Our studies showed neutrophil and humoral immune response are activated in DHF in the LA stage, but the whole immune system was damaged in DHF compared with Dengue Fever (DF), which was a possible reason that leaded to DHF.

Several studies have used multiple genes to distinguish Dengue patients from healthy samples $[39,40]$, DHF from DF [41] and different stages [46]. However, multi-gene lists can limit the sensitivity and specificity of DEGs as disease biomarkers [47, 48]. Compared with previous studies [39-41], our study had several advantages. First of all, this study was based on single gene analyses and these biomarkers were identified, verified and tested in independent datasets through three methods (DEG, WGCNA and ROC) which allowed us to identify a stable and robust biomarker, differing from precious studies only depending on the DEG analysis and analyzed in single dataset. Secondly, we analyzed 22 types of immune cells based on RNA-sequence which contributed to increase understand of the whole immune response during Dengue infection and explore correlations between genes and immune cells. A previous 
study showed that peripheral lymphocyte subset alteration can be independent predictors for clinical characteristics and treatment efficacy of COVID-19 [49]. Interestingly, we found that the fraction of Plasma cells, activated memory CD4+ T cells and Monocytes in Dengue patients can be used to distinguish these clinical stages for Dengue patients. But there is still a shortcoming of this study, because the study only uses public datasets to analyze, verify and test, without clinical verification and test. Our research has provided help in accurately diagnosing Dengue, and will help analyze mechanisms of DHF and benefit to clinical treatment in the future.

\section{Materials and Methods}

\subsection{Data Source}

We obtained gene expression datasets of Dengue patients, including GSE43777 dataset analyzing Two chip types (affymetrix HG-U133 plus 2 in GLP570 platform and HG-Focus in GLP201 platform) [41], GSE28405 dataset [50] and GSE51808 dataset [51] from Gene Expression Omnibus databases (GEO, http://www. ncbi. nlm. nih.gov/geo). All these data were collected from a public and open database (GEO), so ethical review and approval was not required for the study.

Regarding gene expression levels in Convalescent (C) or Early Acute (EA) stages as baselines, these patients were divided into 3 groups, $\mathrm{C}$ vs EA, $\mathrm{C}$ vs Late Acute (LA) and EA vs LA, as precious studies [41]. The Principal Component Analysis (PCA) [52] was used to explore whether different stages can be distinguished clearly. 


\subsection{Differentially Expressed Genes (DEGs) Analysis and Functional Annotation of DEGs} A website (http://sangerbox.com/Tool) based on a R package "Limma" were applied to analyze DEGs among three phases and between DHF and DF for Dengue patients, and a R package "clusterProfiler" in R version 4.1.0 and a GSEA software (4.1.0) [53] were used to performed Gene Ontology (GO) enrichment, Kyoto Encyclopedia of Genes and Genomes (KEGG) analysis and Gene set enrichment analysis (GSEA) to explore potential bio-function and enrichment pathways for DEGs.

\subsection{Weighted Co-Expression Network Analysis (WGCNA) [54]}

A "WGCNA" package was used to identify the module displaying the highest conformity with the development disease of Dengue.

\subsection{Evaluation Immune Cells of the Whole Blood Samples}

we estimated fractions of immune cells in whole blood samples by the "CIBERSORT" website (https://cibersortx.stanford.edu/index.php) which can be used to input gene expression data and then obtained a fraction estimate of 22 immune cell types in whole blood samples [55].

\subsection{Receiver Operator Characteristic Curve (ROC)}

ROC [56] was applied to identify, valid and test value of biomarkers and immune cells in distinguish stages and severity. 


\subsection{Statistical Analyses}

R software (version 4.1.0) and R. Studio (version 1.9.0) were used to analyze data and visualize results. We applied the t-test, Mann-Whitney $U$ test and the chi-square test to identify differences between two groups. Correlation analyses were showed according to the Pearson and Spearman theories. P values $<0.05$ was regarded as statistical significance.

\section{Supplementary Materials:}

Figure S. (A-C) PCA shows that different stages can be distinguished. Weighted co-expression network analysis. (D-F) Showing the cutoff height. (G-I) Sample clustering. (J) Shared gene (CCL2) in the LA stage between DEGs (between DHF and DH) and 232 autophagy-related genes, and (K) its expression levels. Similar expression levels of CD38 and CDKN1C between DH and DHF (L) and among different serotypes (M).
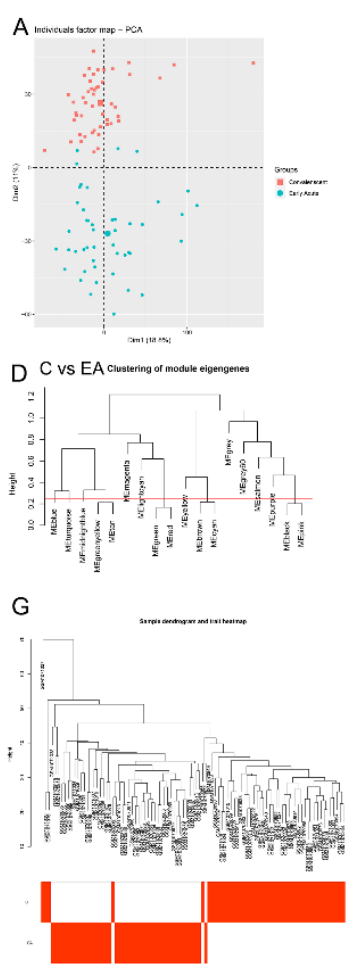
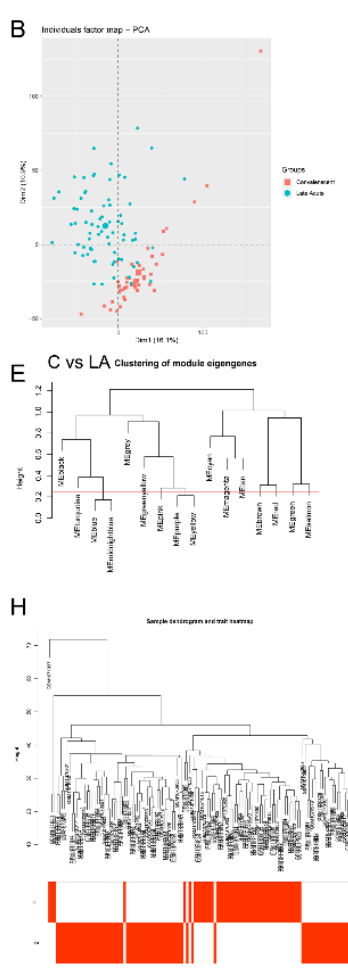
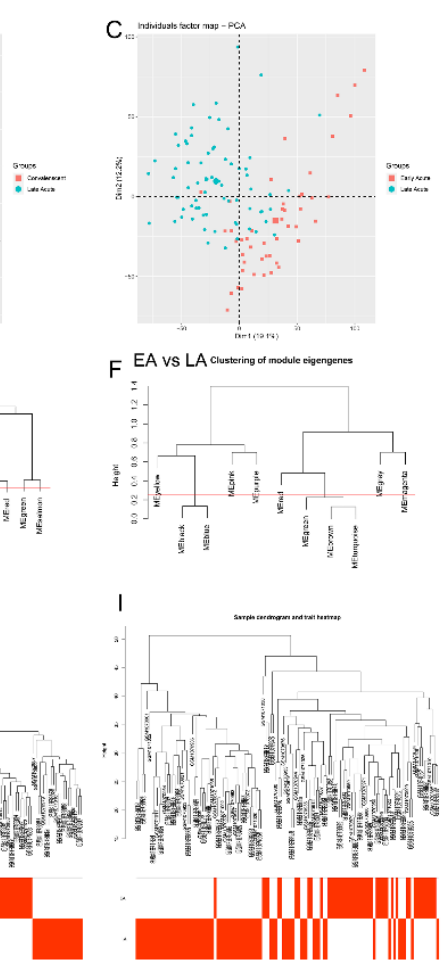
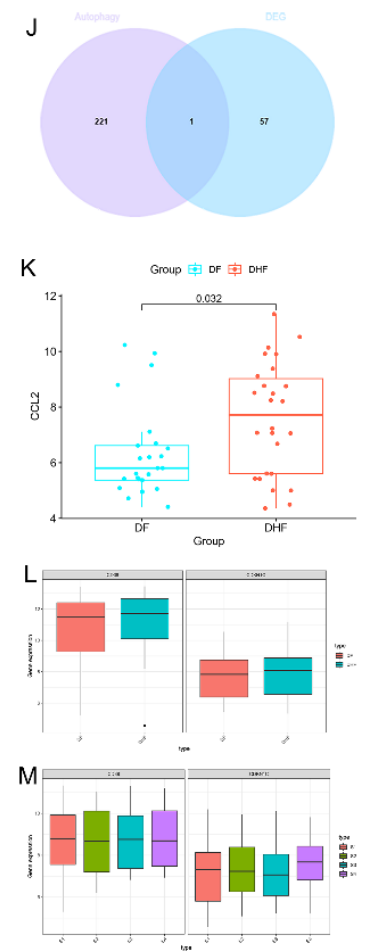

Table S1. DEGs in the C vs EA group

Table S2. DEGs in the C vs LA group 
Table S3. DEGs in the EA vs LA group

Table S4. DEGs from comparing DHF with DF in EA group

Table S5. DEGs from comparing DHF with DF in LA group

Acknowledgments: We would like to thank Mr. Yinde Huang for his support with bioinformatics analyses and pay tribute to the contributions of public databases such as GEO to human medicine.

Disclosure statement: No potential conflict of interest was reported by the author(s).

Ethical approval: This article is not involved in human or animal participants.

Data Availability Statement: The original contributions presented in the study are included in the article/Supplementary Materials, and further inquiries can be directed to the corresponding author/s.

Author contributions: NX designed the analytical strategies, performed data analyses, and wrote the manuscript. QS conceived the research and wrote the manuscript. All authors contributed to the article and approved the submitted version.

Funding: The study was sponsored by the National Natural Science Foundation of China (31970868); the Natural Science Foundation of Yunnan (2019ZF004); Yunnan health training project of high-level talents (H-2017052); Innovation Team Project of Yunnan Science and 
Technology Department [202105AE160020].

\section{References}

1. Z Zhang, J.; Shu, Y.; Shan, X.; Li, D.; Ma, D.; Li, T.; Long, S.; Wang, X.; Pan, Y.; Chen, J.; Liu, P.; Sun, Q., Co-circulation of three dengue virus serotypes led to a severe dengue outbreak in Xishuangbanna, a border area of China, Myanmar, and Laos, in 2019. International journal of infectious diseases : IJID : official publication of the International Society for Infectious Diseases 2021, 107, 15-17.

2. Carbajo, A. E.; Cardo, M. V.; Vezzani, D., Is temperature the main cause of dengue rise in non-endemic countries? The case of Argentina. International Journal of Health Geographics 2012, 11, (1), 26.

3. Hiatt, T.; Nishikiori, N., Epidemiology and control of tuberculosis in the Western Pacific Region: analysis of 2012 case notification data. Western Pacific surveillance and response journal : WPSAR 2014, 5, (1), 25-34.

4. Brady, O. J.; Gething, P. W.; Bhatt, S.; Messina, J. P.; Brownstein, J. S.; Hoen, A. G.; Moyes, C. L.; Farlow, A. W.; Scott, T. W.; Hay, S. I., Refining the global spatial limits of dengue virus transmission by evidence-based consensus. PLoS neglected tropical diseases 2012, 6, (8), e1760.

5. Inizan, C.; Minier, M.; Prot, M.; O'Connor, O.; Forfait, C.; Laumond, S.; Marois, I.; Biron, A.; Gourinat, A. C.; Goujart, M. A.; Descloux, E.; Sakuntabhai, A.; Tarantola, A.; Simon-Lorière, E.; Dupont-Rouzeyrol, M., Viral evolution sustains a dengue outbreak of enhanced severity. Emerging microbes \& infections 2021, 10, (1), 536-544.

6. Thadchanamoorthy, $V_{\text {.; }}$ Dayasiri, K., Postdengue chronic fatigue syndrome in an adolescent boy. BMJ case reports 2021, 14, (6).

7. Kanesa-Thasan, N.; Sun, W.; Kim-Ahn, G.; Van Albert, S.; Putnak, J.; King, A.; Raengsakulsrach, B.; Christ-Schmidt, H.; Gilson, K.; Zahradnik, J., Safety and immunogenicity of attenuated dengue virus vaccines (Aventis Pasteur) in human volunteers. Vaccine 2001, 19, (23-24), 3179-3188.

8. Kitchener, S.; Nissen, M.; Nasveld, P.; Forrat, R.; Yoksan, S.; Lang, J.; Saluzzo, J.-F., Immunogenicity and safety of two live-attenuated tetravalent dengue vaccine formulations in healthy Australian adults. Vaccine 2006, 24, (9), 1238-1241.

9. Guy, B.; Briand, O.; Lang, J.; Saville, M.; Jackson, N., Development of the Sanofi Pasteur tetravalent dengue vaccine: One more step forward. Vaccine 2015, 33, (50), 7100-7111.

10. Osorio, J. E.; Velez, I. D.; Thomson, C.; Lopez, L.; Jimenez, A.; Haller, A. A.; Silengo, S.; Scott, J.; Boroughs, K. L.; Stovall, J. L., Safety and immunogenicity of a recombinant live attenuated tetravalent dengue vaccine (DENVax) in flavivirus-naive healthy adults in Colombia: a randomised, placebo-controlled, phase 1 study. The Lancet Infectious Diseases 2014, 14, (9), 830-838.

11. Martinez, L. J.; Lin, L.; Blaylock, J. M.; Lyons, A. G.; Bauer, K. M.; De La Barrera, R.; Simmons, M.; Jarman, R. G.; Currier, J. R.; Friberg, H., Safety and immunogenicity of a dengue virus 
serotype-1 purified-inactivated vaccine: results of a phase 1 clinical trial. The American journal of tropical medicine and hygiene 2015, 93, (3), 454.

12. Raviprakash, K.; Wang, D.; Ewing, D.; Holman, D. H.; Block, K.; Woraratanadharm, J.; Chen, L.; Hayes, C.; Dong, J. Y.; Porter, K., A tetravalent dengue vaccine based on a complex adenovirus vector provides significant protection in rhesus monkeys against all four serotypes of dengue virus. Journal of virology 2008, 82, (14), 6927-6934.

13. Shukla, R.; Ramasamy, V.; Shanmugam, R. K.; Ahuja, R.; Khanna, N., Antibody-Dependent Enhancement: A Challenge for Developing a Safe Dengue Vaccine. Frontiers in cellular and infection microbiology 2020, 10, 572681.

14. Narayan, R.; Tripathi, S., Intrinsic ADE: The Dark Side of Antibody Dependent Enhancement During Dengue Infection. Frontiers in cellular and infection microbiology 2020, 10, 580096.

15. Bournazos, S.; Gupta, A.; Ravetch, J. V., The role of IgG Fc receptors in antibodydependent enhancement. Nature reviews. Immunology 2020, 20, (10), 633-643.

16. Deng, S. Q.; Yang, X.; Wei, Y.; Chen, J. T.; Wang, X. J.; Peng, H. J., A Review on Dengue Vaccine Development. Vaccines 2020, 8, (1).

17. Malavige, G. N.; Jeewandara, C.; Ghouse, A.; Somathilake, G.; Tissera, H., Changing epidemiology of dengue in Sri Lanka-Challenges for the future. PLoS neglected tropical diseases 2021, 15, (8), e0009624.

18. Eder, M.; Cortes, F.; Teixeira de Siqueira Filha, N.; Araújo de França, G. V.; Degroote, S.; Braga, C.; Ridde, V.; Turchi Martelli, C. M., Scoping review on vector-borne diseases in urban areas: transmission dynamics, vectorial capacity and co-infection. Infectious diseases of poverty 2018, 7, (1), 90.

19. Mizushima, N., A brief history of autophagy from cell biology to physiology and disease. Nature cell biology 2018, 20, (5), 521-527.

20. Kroemer, G.; Mariño, G.; Levine, B., Autophagy and the integrated stress response. Molecular cel/ 2010, 40, (2), 280-293.

21. Chu, L. W.; Yang, C. J.; Peng, K. J.; Chen, P. L.; Wang, S. J.; Ping, Y. H., TIM-1 As a Signal Receptor Triggers Dengue Virus-Induced Autophagy. Int J Mo/ Sci 2019, 20, (19).

22. Lu, Z. Y.; Cheng, M. H.; Yu, C. Y.; Lin, Y. S.; Yeh, T. M.; Chen, C. L.; Chen, C. C.; Wan, S. W.; Chang, C. P., Dengue Nonstructural Protein 1 Maintains Autophagy through Retarding Caspase-Mediated Cleavage of Beclin-1. Int J Mol Sci 2020, 21, (24).

23. Sun, P.; Nie, K.; Zhu, Y.; Liu, Y.; Wu, P.; Liu, Z.; Du, S.; Fan, H.; Chen, C. H.; Zhang, R.; Wang, P.; Cheng, G., A mosquito salivary protein promotes flavivirus transmission by activation of autophagy. Nature communications 2020, 11, (1), 260

24. Heaton, N. S.; Randall, G., Dengue virus-induced autophagy regulates lipid metabolism. Cell host \& microbe 2010, 8, (5), 422-32.

25. Huang, X.; Yue, Y.; Li, D.; Zhao, Y.; Qiu, L.; Chen, J.; Pan, Y.; Xi, J.; Wang, X.; Sun, Q.; Li, Q., Antibody-dependent enhancement of dengue virus infection inhibits RLR-mediated Type-I IFN-independent signalling through upregulation of cellular autophagy. Scientific reports 2016, 6, 22303.

26. Yeo, A. S.; Azhar, N. A.; Yeow, W.; Talbot Jr, C. C.; Khan, M. A.; Shankar, E. M.; Rathakrishnan, A.; Azizan, A.; Wang, S. M.; Lee, S. K., Lack of clinical manifestations in asymptomatic dengue infection is attributed to broad down-regulation and selective up-regulation of host defence response genes. PloS one 2014, 9, (4), e92240. 
27. Simon-Lorière, E.; Duong, V.; Tawfik, A.; Ung, S.; Ly, S.; Casadémont, I.; Prot, M.; Courtejoie, N.; Bleakley, K.; Buchy, P.; Tarantola, A.; Dussart, P.; Cantaert, T.; Sakuntabhai, A., Increased adaptive immune responses and proper feedback regulation protect against clinical dengue. Science translational medicine 2017, 9, (405).

28. OhAinle, M.; Balmaseda, A.; Macalalad, A. R.; Tellez, Y.; Zody, M. C.; Saborío, S.; Nuñez, A.; Lennon, N. J.; Birren, B. W.; Gordon, A.; Henn, M. R.; Harris, E., Dynamics of dengue disease severity determined by the interplay between viral genetics and serotype-specific immunity. Science translational medicine 2011, 3, (114), 114ra128.

29. Garcia-Bates, T. M.; Cordeiro, M. T.; Nascimento, E. J.; Smith, A. P.; de Melo, K. M. S.; McBurney, S. P.; Evans, J. D.; Marques, E. T.; Barratt-Boyes, S. M., Association between magnitude of the virus-specific plasmablast response and disease severity in dengue patients. The Journal of Immunology 2013, 190, (1), 80-87.

30. Duangchinda, T.; Dejnirattisai, W.; Vasanawathana, S.; Limpitikul, W.; Tangthawornchaikul, N.; Malasit, P.; Mongkolsapaya, J.; Screaton, G., Immunodominant T-cell responses to dengue virus NS3 are associated with DHF. Proceedings of the National Academy of Sciences 2010, 107, (39), 16922-16927.

31. Mlera, L.; Offerdahl, D. K.; Dorward, D. W.; Carmody, A.; Chiramel, A. I.; Best, S. M.; Bloom, M. E., The liver $X$ receptor agonist LXR 623 restricts flavivirus replication. Emerging microbes \& infections 2021, 10, (1), 1378-1389.

32. Hong, M.; Tao, S.; Zhang, L.; Diao, L.-T.; Huang, X.; Huang, S.; Xie, S. -J.; Xiao, Z.-D.; Zhang, H., RNA sequencing: new technologies and applications in cancer research. Journal of Hematology \& Oncology 2020, 13, (1), 166.

33. Hill, S. R.; Taparia, T.; Ignell, R., Regulation of the antennal transcriptome of the dengue vector, Aedes aegypti, during the first gonotrophic cycle. BMC Genomics 2021, 22, (1), 71.

34. Azlan, A.; Obeidat, S. M.; Theva Das, K.; Yunus, M. A.; Azzam, G., Genome-wide identification of Aedes albopictus long noncoding RNAs and their association with dengue and Zika virus infection. PLoS neglected tropical diseases 2021, 15, (1), e0008351.

35. Jiang, L.; Ma, D.; Ye, C.; Li, L.; Li, X.; Yang, J.; Zhao, Y.; Xi, J.; Wang, X.; Chen, J.; Pan, Y.; Shan, X.; Sun, Q., Molecular Characterization of Dengue Virus Serotype 2 Cosmospolitan Genotype From 2015 Dengue Outbreak in Yunnan, China. Frontiers in Cellular and Infection Microbiology 2018, 8, (219).

36. Wen, S.; Ma, D.; Lin, Y.; Li, L.; Hong, S.; Li, X.; Wang, X.; Xi, J.; Qiu, L.; Pan, Y.; Chen, J.; Shan, X.; Sun, Q., Complete Genome Characterization of the 2017 Dengue Outbreak in Xishuangbanna, a Border City of China, Burma and Laos. Front Cell Infect Microbio/ 2018, $8,148$.

37. Wang, X.; Ma, D.; Huang, X.; Li, L.; Li, D.; Zhao, Y.; Qiu, L.; Pan, Y.; Chen, J.; Xi, J.; Shan, X.; Sun, Q., Complete genome analysis of dengue virus type 3 isolated from the 2013 dengue outbreak in Yunnan, China. Virus research 2017, 238, 164-170.

38. Jiang, L.; Sun, Q., The Role of Autophagy-Mediated Dengue Virus Antibody-Dependent Enhancement Infection of THP-1 Cells. Intervirology 2020, 63, (1-6), 57-65.

39. Zhong, X. L.; Liao, X. M.; Shen, F.; Yu, H. J.; Yan, W. S.; Zhang, Y. F.; Ye, J. J.; Lv, Z. P., Genome-wide profiling of mRNA and IncRNA expression in dengue fever and dengue hemorrhagic fever. FEBS open bio 2019, 9, (3), 468-477.

40. Xie, L. M.; Yin, X.; Bi, J.; Luo, H. M.; Cao, X. J.; Ma, Y. W.; Liu, Y. L.; Su, J. W.; Lin, G. L.; Guo, 
X. G., Identification of potential biomarkers in dengue via integrated bioinformatic analysis. PLoS neglected tropical diseases 2021, 15, (8), e0009633.

41. Sun, P.; García, J.; Comach, G.; Vahey, M. T.; Wang, Z.; Forshey, B. M.; Morrison, A. C.; Sierra, G.; Bazan, I.; Rocha, C.; Vilcarromero, S.; Blair, P. J.; Scott, T. W.; Camacho, D. E.; Ockenhouse, C. F.; Halsey, E. S.; Kochel, T. J., Sequential waves of gene expression in patients with clinically defined dengue illnesses reveal subtle disease phases and predict disease severity. PLoS neglected tropical diseases 2013, 7, (7), e2298.

42. Heaton, N. S.; Randall, G., Dengue virus and autophagy. Viruses 2011, 3, (8), 1332-41.

43. Chen, T.; Zhang, H.; Liu, Y.; Liu, Y. X.; Huang, L., EVenn: Easy to create repeatable and editable Venn diagrams and Venn networks online. Journal of genetics and genomics = Yi chuan xue bao 2021

44. Castañeda, D. M.; Salgado, D. M.; Narváez, C. F., B cells naturally induced during dengue virus infection release soluble $\mathrm{CD} 27$, the plasma level of which is associated with severe forms of pediatric dengue. Virology 2016, 497, 136-145.

45. Nguyen, H. D.; Chaudhury, S.; Waickman, A. T.; Friberg, H.; Currier, J. R.; Wallqvist, A., Stochastic Model of the Adaptive Immune Response Predicts Disease Severity and Captures Enhanced Cross-Reactivity in Natural Dengue Infections. Frontiers in immunology 2021, 12, 696755.

46. Cougnoux, A.; Dalmasso, G.; Martinez, R.; Buc, E.; Delmas, J.; Gibold, L.; Sauvanet, P.; Darcha, C.; Déchelotte, P.; Bonnet, M.; Pezet, D.; Wodrich, H.; Darfeuille-Michaud, A.; Bonnet, R., Bacterial genotoxin colibactin promotes colon tumour growth by inducing a senescence-associated secretory phenotype. Gut 2014, 63, (12), 1932-42.

47. Deng, M.; Yin, Y.; Zhang, Q.; Zhou, X.; Hou, G., Identification of Inflammation-Related Biomarker Lp-PLA2 for Patients With COPD by Comprehensive Analysis. Frontiers in immunology 2021, 12, 670971

48. Tang, B. M.; Shojaei, M.; Parnell, G. P.; Huang, S.; Nalos, M.; Teoh, S.; O'Connor, K.; Schibeci, S.; Phu, A. L.; Kumar, A.; Ho, J.; Meyers, A. F. A.; Keynan, Y.; Ball, T.; Pisipati, A.; Kumar, A.; Moore, E.; Eisen, D.; Lai, K.; Gillett, M.; Geffers, R.; Luo, H.; Gul, F.; Schreiber, J.; Riedel, S.; Booth, D.; McLean, A.; Schughart, K., A novel immune biomarker IFI27 discriminates between influenza and bacteria in patients with suspected respiratory infection. The European respiratory journa/ 2017, 49, (6).

49. Wang, F.; Nie, J.; Wang, H.; Zhao, Q.; Xiong, Y.; Deng, L.; Song, S.; Ma, Z.; Mo, P.; Zhang, Y., Characteristics of Peripheral Lymphocyte Subset Alteration in COVID-19 Pneumonia. The Journal of infectious diseases 2020, 221, (11), 1762-1769.

50. Tolfvenstam, T.; Lindblom, A.; Schreiber, M. J.; Ling, L.; Chow, A.; Ooi, E. E.; Hibberd, M. L., Characterization of early host responses in adults with dengue disease. BMC infectious diseases 2011, 11, 209.

51. Soares-Schanoski, A.; Baptista Cruz, N.; de Castro-Jorge, L. A.; de Carvalho, R. V. H.; Santos, C. A. D.; Rós, N. D.; Oliveira, Ú.; Costa, D. D.; Santos, C.; Cunha, M. D. P.; Oliveira, M. L. S.; Alves, J. C.; Océa, R.; Ribeiro, D. R.; Gonçalves, A. N. A.; Gonzalez-Dias, P.; Suhrbier, A.; Zanotto, P. M. A.; Azevedo, I. J.; Zamboni, D. S.; Almeida, R. P.; Ho, P. L.; Kalil, J.; Nishiyama, M. Y. J.; Nakaya, H. I., Systems analysis of subjects acutely infected with the Chikungunya virus. PLoS pathogens 2019, 15, (6), e1007880.

52. Wold, S.; Esbensen, K.; Geladi, P., Principal component analysis. Chemometrics \& 
Intelligent Laboratory Systems 1987, 2, (1-3), 37-52.

53. Subramanian, A.; Tamayo, P.; Mootha, V. K.; Mukherjee, S.; Ebert, B. L.; Gillette, M. A.; Paulovich, A.; Pomeroy, S. L.; Golub, T. R.; Lander, E. S.; Mesirov, J. P., Gene set enrichment analysis: A knowledge-based approach for interpreting genome-wide expression profiles. Proceedings of the National Academy of Sciences 2005, 102, (43), 15545-15550.

54. Zhang, B.; Horvath, S., A general framework for weighted gene co-expression network analysis. Statistical Applications in Genetics \& Molecular Biology 2005, 4, (1), Article17.

55. Newman, A. M.; Steen, C. B.; Liu, C. L.; Gentles, A. J.; Chaudhuri, A. A.; Scherer, F.; Khodadoust, M. S.; Esfahani, M. S.; Luca, B. A.; Steiner, D.; Diehn, M.; Alizadeh, A. A., Determining cell type abundance and expression from bulk tissues with digital cytometry. Nature biotechnology 2019, 37, (7), 773-782.

56. Nettleman, M. D., Receiver operator characteristic (ROC) curves. Infection Control \& Hospital Epidemiology 1988, 9, (8), 374-377. 